MACROPHAGES

\title{
Lipids and stress - a deadly duo
}

Acute atherothrombotic vascular disease (the leading cause of death in the industrialized world) is associated with the conversion of small, clinically silent atherosclerotic lesions into necrotic plaques that are prone to rupture. A new study in Cell Metabolism provides a missing link in this conversion, showing that a combination of endoplasmic reticulum (ER) stress and atherogenic lipids or lipoproteins triggers the macrophage apoptosis and plaque necrosis that are necessary for clinical disease.

Previous studies have provided a link between ER stress and the susceptibility of macrophages to apoptosis, but it was not clear what additional pro-apoptotic signals are needed to trigger apoptosis. Here, the authors subjected macrophages to levels of ER stress that are likely to occur in vivo and that do not trigger apoptosis on their own, and showed that apoptosis in these macrophages

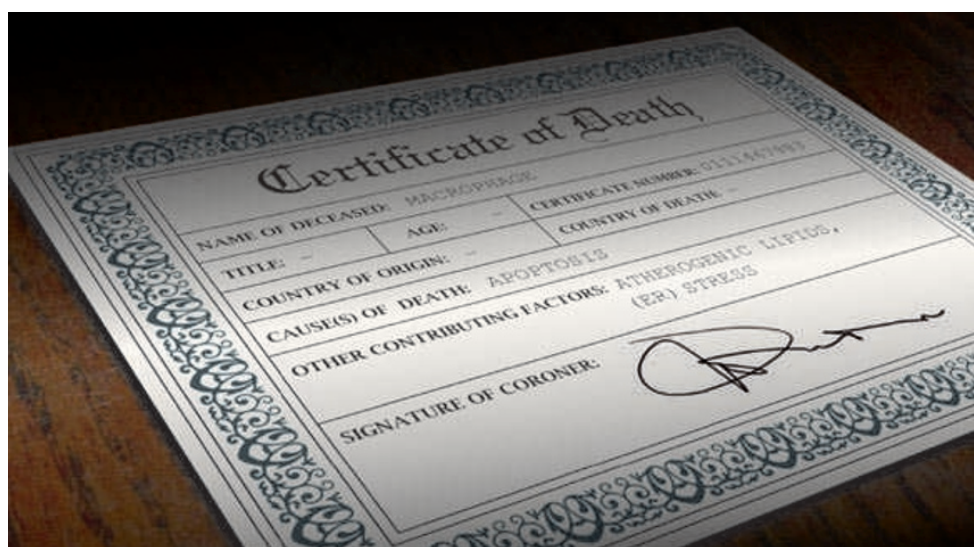

was induced by oxidized low-density lipoprotein (oxLDL) or oxidized phospholipids. The apoptosis was dependent on binding and internalization of oxidized phospholipids by the scavenger receptor CD36. Toll-like receptor 2 (TLR2; which is known to associate with CD36), TLR6 (which forms a heterodimer with TLR2) and the signalling adaptor myeloid differentiation primary response protein 88 (MYD88) were also required for oxLDLinduced apoptosis of ER-stressed macrophages. Indeed, saturated fatty acids that are ligands of TLR2 (such as lipoteichoic acid) could also provide the pro-apoptotic stimulus to CD36-expressing ER-stressed macrophages.

Further investigation of the mechanism of apoptosis revealed that reactive oxygen species (ROS) are generated by NADPH oxidase in macrophages during ER stress.
Accordingly, macrophages lacking the NADPH oxidase subunit NOX2 did not undergo apoptosis following treatment with CD36 ligands and thapsigargin (an ER stress-inducing agent). Further studies showed that extracellular signal-regulated kinase (ERK) activation downstream of TLR2 recruits p47phox (also known as NCF1) to the NADPH oxidase. This promotes ROS production and induction of apoptosis in macrophages that are exposed to lipoteichoic acid and thapsigargin.

The CD36-ER stress apoptosis pathway was shown to be relevant in vivo as macrophages isolated from mice fed a diet of saturated fatty acids and given thapsigargin showed a marked increase in apoptosis compared with controls; a process that was dependent on CD36 and TLR2 expression. Moreover, in fat-fed mice that were deficient for the LDL receptor (a model of atherosclerosis) and transplanted with $\mathrm{Tlr} \mathrm{2}^{-/-} \mathrm{Tlr} 4^{-/-}$ bone marrow, advanced lesions were protected from macrophage apoptosis and plaque necrosis.

Together, these findings identify a role for CD36 and TLR2 in the progression of atherosclerosis, through triggering apoptosis in ER-stressed macrophages.

Lucy Bird

ORIGINAL RESEARCH PAPER Seimon, T. A. et al. Atherogenic lipids and lipoproteins trigger CD36-TLR2-dependent apoptosis in macrophages undergoing endoplasmic reticulum stress. Cell Metab. 12, 467-482 (2010) 